\title{
Mathematical Model of Ship Dynamic Positioning Control System Based on Nonlinear Control Theory
}

\author{
Alian Shi \\ Qilu Normal University, Shandong, Jinan, 250000
}

Keywords: nonlinear control; dynamic positioning; model construction

\begin{abstract}
Dynamic positioning is a kind of positioning system specially designed for ships in marine engineering. It first determines the exact position of the ship through the sonar, and then uses the automatic control system on the ship to issue instructions to the ship to achieve the positioning effect. While positioning, the information transmits via the computer. Most of them require only a few minutes of steady control of the ship being sailed at a designated location. After the positioning system is turned on, the current sensors, wind direction sensors, heading sensors, and position sensors on the ship start working at the same time, and the real-time data are quickly transmitted to the computer. The computer then compares with the originally scheduled position and sends a powerful command to push the ship forward or adjust. The original thrust allows the ship to stop at a specified position accurately and steadily. The system can also use other advanced technologies to control the error within a few meters, and the technology can be used not only for ship positioning but also to control the distance between the ship and the ship affixed to make it safe and reliable. The ship's dynamic positioning system is not restricted by the distance and depth of water. Ships can use this system to perform many offshore operations such as sand dredging, oil extraction, and sea rescue, making it difficult to perform feasible.
\end{abstract}

\section{Introduction}

A ship is a general term for various types of ships. It is a type of marine vehicle that can navigate or berth with a certain area of water for operation or transportation, and has different equipment, technical performance, and structural modes depending on the use requirements or the purpose of work. The positioning of the ship has two meanings. The first level is determined by the navigator to determine its position on the surface of the earth, or the precise position of the ship is obtained without reference to other data; the extra layer is to keep the ship in its designated position. In the middle of the 20th century, marine technology developed rapidly and dynamic positioning systems began to emerge during this period. Dynamic positioning, through the automatic positioning system, allows ships to use their own power to withstand the impact of the sea and other currents on the ship. Dynamic positioning can ensure that the ship does not float up or down through anchor lines, etc., reducing the complex anchoring process, and is not affected by distance, depth, etc. Some of them can even issue commands to ships in advance through changes in the weather direction. The ship dynamic positioning system is another type of positioning method that has practical significance for modern marine operations. This paper takes the dynamic positioning system as being the basis, focusing on the construction of relevant mathematical models under the nonlinear control theory.

\section{Mathematical Model of Ship Dynamic Positioning}

Due to the complexity and non-linearity of the ship's motion at sea, accurate mathematical models of ship dynamic positioning systems are difficult to be established ${ }^{[1]}$. Ships working at sea, due to the impact of external environment waves, wind force, wind direction, etc., will occur six degrees of freedom movement, for the ship's movement at sea, dynamic positioning system used to control the ship's ship and the horizontal and vertical direction of the plane movement. The algebraic model of the ship is divided into high frequency motion and low frequency motion. The initial high-frequency is the motion caused by the first-order wave. It cannot be powered back to the 
control system. The controller we studied cannot control this kind of motion. It does not lead to the horizontal position of the vessel to move, but only the vertical. The direction causes the ship to shake up and down; the second kind of low-frequency is a type of movement caused by the external environment and secondary waves. The dynamic positioning control system we studied is only used to check such movements. Only the subsequent movement, that is, the movement that affects the horizontal position of the ship, is what we want to study. This reduces the energy required to operate the ship and also reduces the production cost of the control system.

With the ship's high frequency motion model, the ship will eventually stay in its initial position due to the back and forth movement of the waves. Theoretically, we do not have to manipulate the high frequency motion of the ship, but in actual measurement, the sensor will also contain a few high frequency motions. About the accuracy of the data, we must establish a model to filter out a few high-frequency motions. The low frequency motion of the ship involves three degrees of freedom of swaying, swaying and boat shaking, which can be described intuitively by establishing a coordinate system. Based on the maneuverability of the ship, a relevant mathematical model for ship operation is established. Finally, the influence of the external environment - wind force, wind direction, wave, current, etc. on the positioning system is studied through the model.

\section{Ship Dynamic Positioning Measurement System}

Different dynamic positioning control systems have separate measurement systems, but measurement systems are indispensable for a single ship. At present, there are many types of position measurement systems, such as GPS positioning systems, sonar positioning systems, and laser positioning systems. Some ships are under a variety of measurement systems that work together. Some shops use a single measurement system. The ship operating area is far from the land, and various positioning systems are not easy to operate in practical applications. Therefore, most ships use GPS positioning systems as measuring equipment. By receiving signals transmitted by satellites, GPS knows the location of satellites, calculates the relative positions of satellites and users, and then determines the position of the receiver itself. Because satellite time and receiver time is not necessarily the same, it is generally necessary to jointly measure four satellites to determine the values Of the four variables XYZ and time. By using a differential method, the measurement error can be reduced and a more accurate value can be achieved. Differential techniques are the observation of one target at two measuring stations, the observation of two targets at one measuring station or the difference between two measurements of one target at one measuring station. This eliminates common errors and gives more accurate positioning values. The wind speed and direction on the sea will also have a great influence on the measurement, so the wind direction anemometer is an important sensor in the measurement process and provides essential reference data for the control of the ship's position. Accurate measurement systems and sensitive sensors work together in order to get the most accurate position of the ship and accurately position the ship.

\section{The Thrust Positioning Control System of the Ship}

The dynamic positioning of a ship means that the disturbance forces and moments of sea winds, waves and other factors shall be counteracted through the thrust and moments generated by the thrusters and propellers to ensure the ship remains relatively stationary in a certain area of the sea[2]. The ship propulsion system and power supply system are two major functional modules. The traditional propulsion system is constituted by the diesel engine and then transmits the energy to the propeller through the propeller shaft to achieve the purpose of propelling the ship. Mostly, three diesel engines constitute the main driving force. The principal driving force is only responsible for pushing the ship forward, and another diesel engine is responsible for driving the generator. The electric propulsion system of the ship uses electric motors to operate the ship's propellers, and at the same time, it integrates electricity for daily use and equipment electricity into a unified whole, which facilitates management and contrasts with the traditional diesel engine as the main driving 
force. Electric propulsion is to propeller the propeller by propelling the motor. It converts mechanical energy into electrical energy through a generator, and then converts electrical energy into mechanical energy to achieve energy transfer. The traditional diesel generator is mainly combined with the power generator.

\section{Position Control Capability of Ship Positioning}

The power positioning and positioning capability system of the ship are an important component of the positioning system, which means that the ship applies its own propulsion system to obtain the maximum thrust. At the same time, $d$ it can resist the forces of wind waves and currents to achieve a better positioning effect. Off-line positioning ability of the ship refers to the ability of a ship to control a position when the ship is completely normal in all aspects such as propulsion. And the off-line control capability of a ship refers to the ability of the ship to control its position when there is a problem with the propulsion system or other systems. The ship control capability analysis system is of great importance to the ship. Many laboratories in foreign countries have made significant research work on this system. Related research companies and universities have come forward with power system control capacity calculation software that provides us with scientific evidence.

First of all, we need to establish a model of force acting on ships, such as storm waves and sea currents on the sea level, and then establish models for calculation of coefficient of non-dimensionality forces acting on ships, such as wind waves and sea currents, and then build a propeller thrust distribution model according to the propulsion system configuration scheme. By constructing the genetic operators of genetic algorithms, we can obtain the calculation methods for off-line ship control capability and on-line control capability. The ship positioning system is an extremely complex control system, and the dynamic positioning control system is an inseparable part. We must establish the power requirements of the thruster before we study its control capacity. If the power is not sufficient, the accuracy of the position data cannot be ensured. Only when the power of the propeller is taken into account, the ship will remain fixed under the influence of the external environment. Its control capabilities can also be evaluated. Ship position control system provides the possibility of precise positioning of the ship and has a very high practical application value.

\section{Conclusion}

The ocean contains new energy, mineral resources and new typesof marine life. It can achieve th e sustainable development of human social resources, and huge marine wealth awaits us to tap. We need to rationally develop and utilize the oceanic areas so that they can play a role in social development and human economic development. With the development of modern seagoing research, our demand for marine resources and energy continues to increase, and the scope of activities has gradually tended to deeper seas. Whether it is fishing at sea or under the sea, ships canbe inextricably linked to ships. The dynamic positioning system of ships has gradually become an indispensable support system. The ship positioning system emerged in the middle of the last century has gradually developed into a mature system equipment. The early analog controllers and the digital computer control systems that have emerged with the development of computer technology have more and more accurate data and have been running for longer. Nowadays, dynamic positioning control systems have become more sophisticated, complex, accurate and reliable. The future world is a brand-new world that belongs to the ocean field.

\section{References}

[1] Shan Zhang, Zaijian Zou. Simulation of Ship Dynamic Positioning Algorithm Based on Fading Memory Adaptive Filter. Ship Mechanics 2017, 21 (12)

[2] Xingxia Niu, Xiaodan Zhang. Application of Nonlinear Backstepping Algorithm in Ship 
Dynamic Positioning System Control. Ship Science and Technology. 2017 (20): 34-36 